\title{
CONCLUSION GÉNÉRALE COMMENT, DE 1643 À 1756, LES FRANÇAIS VOYAIENT-ILS L'EMPIRE?
}

\section{L'intérêt général du problème de la perception d'autrui}

à l'époque moderne: le caractère exemplaire de l'étude de la connaissance du Saint-Empire en France

Roger-Pol Droit s'interroge - on ne saurait que trop saluer une telle démarche -, dans la »chronique « littéraire du »Monde «l, à propos du fait que nombre d'évolutions récentes dans la pensée philosophique transalpine seraient passées inaperçues en France sur l'état réel des connaissances dont nous disposons concernant l'étranger le plus proche, et ce à l'ère de moyens de communication rapides et sophistiqués: „On nous le chante, dit-il, sur tous les tons: nous voilà réellement sur le point de devenir >globaux< [...]. Pourtant, rien ne semble demeurer plus étranger que nos voisins les plus proches, rien ne persiste à être si opaque à notre compréhension que les autres cultures, dans leur cohérence propre et leur diversité. Sous la grande pellicule informatique [...] de puissantes barrières demeurent entre les esprits«. Et de conclure: »Derechef, contrairement à ce qu'on répète à l'envi, il faut donc soutenir cette évidence: le monde n'est pas du tout connu «.

Nous avons pu remarquer que les XVII ${ }^{e}$ et XVIIJe siècles disposaient déjà de médias suffisants pour s'informer sur les pays limitrophes, et l'on peut constater de ce que les progrès des supports de l'information que l'humanité a connus depuis le XVIIle siècle, s'ils ont facilité l'accès au savoir, ne sont pas à l'origine d'un quelconque >désenchantement du monde. L'attention que le journaliste porte de nos jours au problème de la connaissance du voisin démontre cependant l'intérêt d'une étude de cette question dans le passé, démarche qui peut éclaircir le débat actuel en lui redonnant sa profondeur historique.

Les Français de l'Ancien Régime disposaient de nombreux livres imprimés en latin et, surtout depuis le XVIII e siècle, en français pour s'instruire sur l'Allemagne en général et son droit public en particulier: histoires d'Allemagne, histoires et traités systématiques de son droit d'État, récits de voyage, etc. À part les livres, les périodiques, notamment la »Gazette de France $\ll$, renseignaient leurs lecteurs sur les événements politiques et militaires en Allemagne et en Europe centrale, mais, en dépit de la très bonne formation de certains collaborateurs, ne transmettaient que peu d'informations sur le droit constitutionnel de l'Empire en particulier; néanmoins, des événements cruciaux, tels que la conclusion des traités, les élections impériales, certaines délibérations ou décisions de la diète retenaient l'intérêt des rédacteurs. C'est que l'objectif de la presse périodique était d'infor-

I Roger-Pol DroIT, article »Cuisiner l'âme à l'italienne«, dans: Le Monde des livres (12 juillet 1997), p. VI. 
mer des événements, non pas d'analyser les structures constitutionnelles. Par contre, les périodiques littéraires, notamment le »Journal des Savants«, consacraient de nombreux et longs articles aux publications françaises et aux publications latines des auteurs allemands sur le droit public du Saint-Empire. Outre l'imprimé, des textes manuscrits servaient à répandre des connaissances sur la Constitution impériale: les dépêches des envoyés français en Allemagne, la correspondance entre les savants français et leurs confrères allemands, etc. À ces sources d'informations s'ajoutaient les relations personnelles qui existaient entre les diplomates et entre les savants des deux pays. Certaines institutions, comme l'académie de Torcy et, à la fin de notre période, l'école diplomatique de Strasbourg favorisaient la diffusion de connaissances en matière de droit public allemand. Les Alsaciens étaient des intermédiaires essentiels. Par contre, les écrits de propagande (brochures, tracts, etc.) ne poursuivaient pas le but de renseigner et d'informer, mais surtout d'influencer l'opinion; pour cette raison, ces sortes de publications ne traduisaient pas forcément les véritables points de vue de leurs auteurs - toujours est-il qu'en brossant une image plutôt négative de l'empereur et en minimisant ses pouvoirs constitutionnels, elles ne sont apparemment pas restées sans influence sur l'image de l'Empire qui s'est ensuite forgée.

Moins sophistiqués que de nos jours, les médias dont les Français disposaient sous l'Ancien Régime étaient donc tout à fait suffisants pour que l'on pût se faire une image précise et exacte de l'ordre politique et du droit du Saint-Empire. C'est cependant l'intérêt qui est à l'origine de toute connaissance, et non les médias, qui ne sont, comme l'exprime l'étymologie du mot, que des moyens. L'intérêt des Français pour le droit public allemand aux XVII et XVIII ${ }^{e}$ siècles connaît des origines et des formes multiples.

Les résultats de nos recherches, initialement étendues à la période antérieure à 1648, montrent qu'avant la paix de Westphalie on ne s'était pas occupé en France des problèmes du droit public allemand d'une manière aussi globale et continue qu'on ne le fit après cette date. Certes, il y eut de nombreux moments où une situation politique particulière (par exemple, celles qui se présentaient en 1519 et en 1552) incitait les Français à réfléchir sur des questions du droit d'État de l'Allemagne; mais cela se faisait d'une manière plus ou moins intense, dans le cadre d'une problématique politique ou d'une réflexion juridique précises, et concernait surtout des sujets bien délimités, tels que les élections impériales et les questions liées à la légitimité de la résistance contre le souverain.

Il convient néanmoins de constater un intérêt grandissant à l'égard de l'Empire dans la deuxième moitié du XVIIe siècle. Il semble que l'Alsace, la politique des Réunions et généralement la politique étrangère active de Louis XIV dans l'Empire ne soient pas étrangères à ce phénomène. La France a besoin de publicistes versés dans le droit public allemand. Dans un premier temps, elle les trouve surtout en Alsace et en Allemagne. Jean Heiss et Ulric Obrecht propagent le droit public du Saint-Empire en France. L'»Histoire de l'Empire« de Jean Heiss restera le point de référence pour les Français s'intéressant au droit allemand pendant un demi-siècle, des années 1680 aux années 1730. Des ouvrages de référence, 
comme le dictionnaire de Moréri, en ont copié certains passages presque littéralement. Les Alsaciens continuent à jouer un rôle très important au cours du XVIII ${ }^{e}$ siècle. Vers le milieu du siècle, Chrétien-Frédéric Pfeffel publie un »Abrégé chronologique de l'histoire et du droit public d'Allemagne «, qui connaît cinq éditions avant la Révolution. Strasbourg est le haut lieu des études germaniques en France. C'est là qu'enseigne le célèbre historien Schœpflin, auquel succéda son élève Christophe-Guillaume Koch. Au milieu du siècle, nous voyons des Allemands écrire en français sur le droit public du Saint-Empire. Ils ne s'adressent pas exclusivement à un public français. Scheid vise en particulier les jeunes princes des cours allemandes, qui ont pris l'habitude de parler français, cette langue ayant, dans le domaine temporel, pris le relais du latin.

Â partir de la paix de Westphalie, les Français portèrent un intérêt continuel et global à la Constitution du Saint Empire romain germanique. Pour cela, il y avait des raisons politiques, que nous venons d'esquisser, et culturelles. D'abord, la France prit au sérieux le rôle de garant de la Constitution westphalienne qui lui revenait en vertu des traités de Westphalie. En outre, depuis au moins 1635, elle s'était engagée dans une politique active à l'égard du Saint-Empire, que les règlements mêmes des traités de paix éternisèrent puisqu'ils ne permirent point à la France de se désintéresser désormais des affaires de l'Allemagne - nous pensons en particulier au mélange des droits français et allemands qui en résultait en Alsace. Or, à part cette continuité d'intérêts politiques, il y avait d'autres raisons qui n'avaient pas toujours d'effets durant toute la période qui va de 1648 à 1756; parmi ces raisons, que nous avons évoquées au cours de ce livre, on peut par exemple noter l'intérêt que certains protestants français portèrent, au XVII ${ }^{\mathrm{e}}$ siècle, à la sûreté que la Constitution impériale procurait aux protestants allemands, ou le modèle d'organisation politique ${ }^{2}$ que le Saint-Empire fut, au XVIII ${ }^{\text {siècle, }}$ pour certains philosophes français.

Malgré certaines continuités qui ont marqué toute la période que nous avons étudiée, on ne peut donc pas expliquer l'intérêt que les Français portèrent au Saint-Empire par une raison unique. Cette hétérogénéité des intérêts explique aussi la diversité d'opinions qu'on peut détecter dans leurs écrits à l'égard de l'Allemagne. C'est pourquoi, si ce livre est intitulé »La connaissance du Saint Empire en France de la paix de Westphalie au Renversement des alliances«, l'emploi du singulier ( $l a$ connaissance) n'est pas destiné à suggérer une quelconque uniformité de la pensée: en effet, si une partie des Français considéraient l'Empire comme une monarchie, d'autres ne le tenaient même pas pour un >État $<$, mais pour une confédération de souverains indépendants; l'absence d'uniformité ne veut pourtant point dire absence d'unité, parce que les différentes idées qu'on peut saisir en France sur la nature du gouvernement du Saint-Empire s'intègrent dans les courants intellectuels de l'époque et évoluent en fonction d'un transfert et d'une

${ }^{2}$ Pour des réflexions critiques sur ce concept, cf. Georg SCHMIDT, Das frühneuzeitliche Reich - Sonderweg und Modell für Europa oder Staat der deutschen Nation?, dans: SCHNETTGER (dir.), Imperium Romanum, p. 247-277. 
critique mutuels constants; elles s'inspirent, en particulier, des évolutions dans l'Empire et de sa jurisprudence. On peut même assister à une sorte d'histoire croisée précoce, le »Journal des Savants « rendant compte des publications en latin sur l'histoire et le droit de l'Allemagne, et les »Göttingische Gelehrte Anzeigen« et d'autres périodiques allemands commentant les livres parus en français sur ces sujets.

L'étude que nous avons entreprise s'insère par ailleurs, non seulement dans un courant de discussions médiatiques sur le rôle de l'information, mais, circonstance plus importante à notre avis, dans une réflexion historique plus large sur l'Europe des diètes, dont les Constitutions furent malheureusement trop souvent méprisées par les historiographies française et allemande, au XIX ${ }^{\mathrm{e}}$ siècle et dans la première moitié $\mathrm{du} \mathrm{XX}^{\mathrm{e} 3}$, au profit de la recherche, bien compréhensible d'ailleurs, des racines de l'État moderne dans l'absolutisme ${ }^{4}$, qui est, toutefois, un modèle »dont on a peut-être tracé un portrait trop exclusif et trop universel ${ }^{5}$. L'Europe des diètes, comme l'a démontré Fisera, connaît elle aussi ses prolongements dans le monde contemporain ${ }^{6}$. Or, ce débat scientifique est d'actualité lui aussi, comme l'a rappelé Yves Durand: »Les débats actuels, souligne-t-il, dans le cadre européen et français, à propos des rapports contradictoires entre centralisme et décentralisation, trouvent comme leur écho dans les affrontements des XVII et XVIII siècles « ${ }^{7}$. Ce point de vue demeure valable aujourd'hui.

Si la recherche de l'actualité n'est pas notre souci, le caractère de modèle historique que prend, dans ce contexte, la Constitution impériale peut bien être un motif qui incite à centrer la réflexion sur le problème de la perception du Saint-Empire par les Français aux XVII et XVIII' siècles. Or, justement, le Saint-Empire fut déjà un modèle d'organisation politique au XVIII ${ }^{e}$ siècle, comme l'a très bien montré Olaf Asbach dans plusieurs de ces études, un modèle dont s'inspirèrent, entre autres, des théoriciens de l'État et des philosophes français tels que l'abbé de Saint-Pierre et Jean-Jacques Rousseau ${ }^{8}$.

${ }^{3}$ Cf. OfFEnBECK, Der Dreißigjährige Krieg.

${ }^{4}$ Pour l'importance des états provinciaux en France, qui a été traditionnellement sous-estimée, cf. Marie-Laure LEGAY, Les États provinciaux dans la construction de l'État moderne aux XVII ${ }^{\mathrm{e}}$ et XVIII ${ }^{\mathrm{e}}$ siècles, Genève 2001 (Travaux du Grand Siècle, 20).

5 TOLLET, avant-propos, p. 10; cf. aussi: AsCH, DUCHHARDT (dir.), Absolutismus.

${ }^{6} \mathrm{Cf}$. V. Cl. FISERA, Rétrovision: principe parlementaire, exécutif fort et passions nationalistes en Europe centrale, aux époques moderne et contemporaine, dans: TOLLET (dir.), L'Europe des diètes, p. 289-298; cf. aussi Karl BosL (dir.), Der moderne Parlamentarismus und seine Grundlagen in der ständischen Repräsentation. Beiträge des Symposiums der Bayerischen Akademie der Wissenschaften und der International Commission for Representative and Parliamentary Institutions auf Schloß Reisensburg vom 20. bis 25. April 1975, avec le concours de Karl MöckL, Berlin 1976; Bernd GrzeszIck, Vom Reich zur Bundesstaatsidee. Zur Herausbildung der Föderalismusidee als Element des modernen deutschen Staatsrechts, Berlin 1996 (Schriften zum Öffentlichen Recht, 705).

7 Y[ves] DuRAND, préface, dans: TOLLET (dir.), L'Europe des diètes, p. 11-14, ici p. 11.

8 Cf. notamment Olaf AsBaCH, Von der »Union Germanique « zur »Union Européenne «. Historische, philosophische und politische Dimensionen des Föderationsgedankens beim Abbé de Saint-Pierre, dans: HöPEL (dir.), Deutschlandbilder, p. 93-117. 
Après une interruption de cette tradition, non seulement en France, mais aussi en Allemagne, où le Saint-Empire n'a guère servi de référence politique positive entre 1806 et les années 1950, l'historiographie allemande a, pour sa part, redécouvert, après la Seconde Guerre mondiale, les origines de l'État de droit moderne dans les institutions politiques et judiciaires du Saint Empire romain germanique. Ces dernières années, certains historiens, comme Johannes Burkhardt, sont même allés jusqu'à proposer le Saint-Empire moderne comme un modèle pour la construction européenne ${ }^{9}$. On peut s'interroger sur l'utilité d'un tel modèle et sur la pertinence de la comparaison entre le Saint-Empire et l'Europe, cette dernière étant, à la différence du Saint-Empire, une confédération d'États souverains et indépendants.

Mais Burkhardt a raison de souligner que le Saint-Empire peut à juste titre passer pour un >État< plus moderne qu'on n'a voulu le suggérer aux $\mathrm{XIX}^{\mathrm{e}}$ et $\mathrm{XX}^{\mathrm{e}}$ siècles, et qui était plus complexe que les monarchies d'Europe occidentale, qui ont longtemps été considérées comme les véritables précurseurs des États contemporains ${ }^{10}$. Pourtant, au lieu d'échanger un modèle contre l'autre, et d'encenser le Saint-Empire après s'être agenouillé devant l'absolutisme français, on devrait, à notre avis, plutôt tenir compte de la relativité de tout modèle historique.

L'apport principal de notre propre étude est justement, à notre avis, d'avoir montré que non seulement dans l'historiographie postérieure à 1806 , mais aussi dans la perception du Saint-Empire par les contemporains, l'image de l'Empire ne fut ni uniforme ni statique, mais évolua en relation avec les points de vue et les préoccupations des observateurs. Cette image fut élaborée au cours d'un échange constant entre Français et Allemands.

\section{Les cinq conclusions principales de cette étude}

En ce qui concerne les résultats concrets de notre étude, nous pouvons tirer cinq conclusions essentielles de l'examen des sources de la période 1643 à 1756:

En premier lieu, nous avons pu constater que les hommes d'État, les diplomates, les historiens et les juristes français avaient des connaissances plus précises et

9 Cf. BuRKHARDT, Das Reformationsjahrhundert.

${ }^{10}$ Burkhardt oppose ici le compromis constitutionnel entre les compétences des États territoriaux et celles du Saint-Empire comme État fédéral (compromis qu'il qualifie de "synthèse d'avant-garde «) à la formation d'États moins complexes et plus uniformes en Europe occidentale (»avantgardistischen Synthese des Reiches zu den einseitigen und minder komplexen Staatsbildungswegen «), cf. ibid., notamment p. 138. Dans ce livre, Burkhardt parle aussi du système politique du Saint-Empire, qui combine les compétences des États territoriaux avec celles de l'État fédéral, en tant que compromis d'une importance fondamentale pour l'histoire universelle, et qui perdure, d'après lui, dans l'Union européenne (»[das] politische System [des Reiches] mit einzelstaatlichen und bundesstaatlichen Zuständigkeiten [als] einen weltgeschichtlichen Kompromiß [...], der in der deutschen Geschichte erstmals gelungen ist und bis heute fortwirkt, und der in der Europäischen Union besondere Bedeutung erlangt $\ll$, ibid.). 
plus exactes en matière de droit public allemand que l'historiographie l'a supposé avant les recherches de Jürgen Voss et de Klaus Malettke, qui sont à l'origine de la révision de ces opinions traditionnelles.

Deuxièmement, au XVIIe siècle, une terminologie particulière se développa dans la langue française, qui fut propre à décrire d'une manière adéquate les institutions, les réalités juridiques et le fonctionnement de la Constitution de l'Empire, même si elle ne fut pas parfaite en tout point et qu'elle fut loin d'être sans équivoque à tous les égards.

Troisièmement, dans le débat français sur la Constitution de l'Empire, Bodin en ce qui concerne sa vision du Saint Empire romain germanique - jouait un rôle certes non négligeable mais pas pour autant essentiel ${ }^{11}$. Les idées de Bodin, qui interpréta l'Empire comme une aristocratie, tout en n'accordant pas une entière liberté et indépendance aux états représentés à la diète, peuvent être détectées aussi bien avant qu'après 1648 , et surtout à partir de 1740 , mais, à en juger par les sources que nous avons étudiées, elles n'ont point prévalu dans le débat en France à l'époque qui va de la paix de Westphalie au Renversement des alliances. Ce résultat est d'autant plus considérable que la discussion des thèses de Bodin marqua justement le début de la Reichspublicistik, de la science du droit public en Allemagne.

Quatrièmement, on peut noter que l'interprétation de la Constitution du SaintEmpire était l'objet de vives controverses, non seulement dans l'Allemagne même, mais aussi en France. Cela contredit l'opinion qui s'est vulgarisée surtout depuis le $\mathrm{XIX}^{\mathrm{e}}$ siècle pour dominer l'historiographie jusqu'au début des années 1980, selon laquelle une image monolithique de l'ordre constitutionnel de l'Empire aurait prévalu en France à l'époque moderne. Par contre, les Français d'Ancien Régime suivaient de près la controverse qui avait lieu dans l'Empire même, au sujet de l'explication de la Constitution impériale; c'est pourquoi, en principe, on peut dégager, dans la France d'Ancien Régime, les mêmes écoles de pensée qui dominèrent le débat constitutionnel, politique et juridique dans l'Allemagne même. Durant la seconde moitié du XVIIre siècle, c'est l'école du status mixtus qui prévalut en France; cette école voyait dans l'Empire un >État< fédéral qui combinait des éléments monarchiques et aristocratiques. À cette période, la plupart des auteurs ne tendirent point à minimiser l'autorité de l'empereur; nombreux furent ceux qui attachèrent une grande importance aux éléments monarchiques dans le système politique impérial. Toujours est-il qu'à côté de ce discours juridique, et parfois malgré lui, on peut dégager un discours historiographique qui se fonde sur l'idée d'une décadence de l'Empire et de l'autorité impériale depuis la fin de la dynastie carolingienne, et surtout depuis le grand interrègne du XIII siècle. À part ces discours juridique et historiographique, une troisième grille de discours qui faisait partie du débat politique proprement dit, mais qui avait bien des retom-

11 Il n'en va pas de même pour sa théorie de la souveraineté, dont nous ne cherchons en aucune manière à minimiser l'importance, même si tous les auteurs n'ont pas repris son acception de ce concept. 
bées dans les æuvres historiographiques et dans les ouvrages juridiques, soulignait le danger imminent d'une dérive autoritaire du système politique du SaintEmpire; non seulement Charles Quint et Ferdinand II, mais aussi Léopold ler et encore Charles VI passaient pour des empereurs capables de transformer le SaintEmpire en monarchie absolue.

Par ailleurs, certains auteurs n'hésitaient point à combiner ces différentes grilles d'explication, et n'y voyaient apparemment aucune contradiction. C'est que, pour de nombreux auteurs, l'état idéal de l'Empire consistait dans une harmonie entre, d'une part, des États territoriaux jouissant d'une très large autonomie et, d'autre part, l'empereur, qui, lui, devait assurer une certaine unité et une certaine protection de l'Empire; pour ces mêmes auteurs, l'état réel était marqué par la tendance de l'empereur à transgresser ses pouvoirs constitutionnels, en principe limités, qui risquait, selon eux, de détruire cette harmonie. Dans cette perspective, les limites entre la propagande politique et l'historiographie sérieuse ne sont pas toujours bien tracées. La justification de la politique allemande des rois de France en tant que »défenseurs de la liberté germanique «12 n'était pas seulement l'objet de la propagande, mais faisait aussi partie de l'historiographie sérieuse. Cette même conception qui faisait du roi de France le défenseur de la liberté des états d'Empire et de l'empereur une sorte de `tyran`, cherchant à subjuguer les états et à les dépouiller de leurs droits constitutionnels, se retrouvait aussi à la base des écrits de Jean-Yves de Saint-Prest; celui-ci était, entre autres fonctions, chargé de dispenser des cours à l'académie de Torcy et a laissé des mémoires au dépôt des archives, de sorte que l'on doit supposer que sa vision du Saint-Empire a exercé une influence non négligeable sur celle des diplomates français du début du règne de Louis XV.

Au XVIII siècle, et plus particulièrement depuis les années 1740 , on peut assister à un véritable éclatement de toute vision homogène du Saint Empire romain germanique: les idées qu'on peut détecter chez les différents auteurs d'ouvrages imprimés et de manuscrits historiographiques, juridiques et diplomatiques vont de la classification de l'Empire comme monarchie limitée, parfois avec un empereur dont on tend même à surestimer les compétences, dans la tradition du XVII siècle, à la remise en cause du caractère même d'État, que certains auteurs refusent d'accorder au Saint-Empire, le tenant pour une confédération d'États souverains et indépendants.

En cinquième et dernier lieu, une étude comparative des regards européens nous a permis de dégager le fait que les auteurs qui écrivaient en français sur le droit public allemand s'inscrivaient plus largement dans un débat européen et que leurs écrits faisaient partie intégrante d'un discours supranational.

En revanche, il faut noter des différences entre l'analyse interne, en particulier dans les documents du Quai d'Orsay, et l'image de l'Empire dans les écrits de

12 Cette expression est utilisée dans de nombreuses brochures et aussi dans des ouvrages historiographiques, depuis le XVI siècle et encore après 1648 , ainsi que les exemples cités l'ont montré. 
propagande en langue française, par exemple dans certains tracts et brochures que nous avons étudiés et qui dressent surtout un bilan plus négatif de la condition juridique de l'empereur que les documents diplomatiques. La vision de l'Empire qui se dégage à travers l'analyse de tracts et de brochures est sensiblement différente de celle que nous avons pu retrouver dans la plupart des livres imprimés et surtout dans les documents manuscrits du dépôt des archives des Affaires étrangères. Cette vision est plus critique vis-à-vis de l'empereur, plus favorable aux états, dans ses prises de position concrètes comme dans ses conceptions de la Constitution idéale de l'Allemagne, et idéalise somme toute la prétendue liberté originelle des ordres dans une espèce de confédération aristocratique. L'image en noir et blanc que cette littérature donne, d'un côté, d'un empereur qui ne semble que troubler le jeu et miner le bon fonctionnement de la Constitution de l'Empire par ses aspirations à la monarchie universelle, en collaboration avec l'Espagne, ou qui vise, du moins, à l'établissement de la monarchie en Allemagne, et, d'un autre côté, des ordres n'aspirant qu'à la conservation de leurs bons vieux droits, dans le seul but d'assurer le bonheur et le respect des prétendus véritables intérêts de l'Empire, cette image, disions-nous, semble bien être due aux objectifs de cette catégorie de sources dont le but n'est pas de fournir une analyse impartiale ou fiable de la situation politique et constitutionnelle. De là, il paraît bien difficile de tirer des conclusions à propos des véritables conceptions que ces auteurs ont eues de l'Empire; cette catégorie de documents est donc plus propre à montrer comment l'imaginaire sur l'Empire fut instrumentalisé, mais moins apte à reconstituer cet imaginaire lui-même.

Le langage employé dans les différentes catégories de sources que nous avons étudiées est sensiblement hétérogène. Les livres imprimés destinés à un public plus large n'utilisent pas toujours la même terminologie que les documents internes réservés aux seuls spécialistes. Le langage du milieu du XVIIIe siècle est plus >scientifique $<$, pour ainsi dire, que le langage du XVIIe, qui, non seulement dans les tracts, mais aussi dans bon nombre de livres imprimés et documents manuscrits, garde un caractère qu'on pourrait qualifier, dans une certaine mesure, de pamphlétaire ${ }^{13}$. Toujours est-il que nous ne tenons pas la distinction de plusieurs »discours« comme une manière propre à bien décrire la pluralité linguistique et conceptuelle, car une telle différenciation trop nette risquerait de remettre en cause ou même de faire disparaître les points communs, les processus d'interaction et d'osmose ainsi que les affinités d'interprétations qui ont pourtant bien existé, tout en insinuant qu'un »discours « particulier eût été plus homogène qu'il ne l'était effectivement.

De plus, comme on vient de le souligner, on ne peut pas toujours tracer les limites entre la propagande et l'étude sérieuse du droit et de l'histoire d'Allemagne, en particulier chez les auteurs de la deuxième moitié du XVII siècle. Pourtant,

${ }^{13}$ En revanche, même les tracts et les brochures qui paraissent au milieu du XVIII ${ }^{e}$ siècle emploient parfois des expressions plutôt techniques, ce qui atteste l'essor de l'étude de la politique et de la philosophie du droit au siècle des Lumières. 
vers la fin du XVIIe siècle et surtout au XVIII ${ }^{e}$, les ouvrages imprimés tendent à acquérir un caractère scientifique. Les auteurs citent les lois et les autorités qu'ils ont consultées en marge du texte ou dans des notes de bas de page, procèdent à un examen plus critique de leurs sources (par exemple en confrontant des opinions divergentes), et la précision augmente, ainsi que le volume de leurs ouvrages, qui peut atteindre jusqu'à onze tomes. Cependant, les écrits polémiques ne disparaissent point et connaissent même un certain renouveau après 1740 .

Bref, selon les résultats de nos recherches, on peut conclure que la majorité des Français qui pouvaient avoir une certaine connaissance de l'Allemagne a considéré, jusqu'en 1740 environ, l'Empire comme un État fédéral, mais qu'à partir de 1740 le nombre et l'influence de ceux qui le regardaient comme une confédération d'États indépendants ont eu tendance à augmenter; cette évolution n'entraina pas directement ni immédiatement un changement fondamental de la politique française à l'égard de l'Empire, mais elle contribua à jeter les bases successivement d'une attitude vis-à-vis de l'Allemagne puis d'une politique allemande qui allèrent jusqu'à envisager la dissolution du Saint Empire romain germanique, et provoquèrent finalement sa chute. Pourtant, il faut bien voir qu' une telle politique ne fut pas encore sérieusement envisagée en 1756 . On commence alors à entrevoir les racines d'une pensée plus radicale qui remet en cause l'existence même du Saint-Empire, mais les conséquences de cette attitude sont encore virtuelles.

\section{Le droit et les langues de l'Empire dans la culture des diplomates et des élites en France}

Ces dernières années, l'historiographie s'est interrogée sur l'importance du Renversement des alliances. Fut-ce vraiment une rupture, un changement d'époque? Nous nous abstiendrons de discuter cette question comme problème d'histoire politique $^{14}$. Mais il nous paraît indubitable que les évolutions politiques des années 1740 et 1750 ont eu des répercussions notables sur la vision française du SaintEmpire. Traditionnellement, depuis les temps de Maximilien Ier et de Charles Quint, l'antagonisme dynastique ${ }^{15}$ avait conditionné l'image que l'on s'était faite de l'empereur comme adversaire dont il avait été nécessaire de limiter les pouvoirs au profit des états, la fameuse liberté germanique ayant servi de contrepoids, destiné à contrebalancer l'autorité de l'empereur. Cette perception avait échappé au domaine politique proprement dit pour se manifester dans l'historiographie et dans les écrits juridiques des auteurs français. Le fait que l'empereur ne fût plus l'adversaire principal de la France dans l'Empire, à partir de 1756 et déjà entre 1742 et 1745 , fut comme une libération d'un vieux préjugé.

${ }^{14}$ Pour ce problème, cf. BrauN, Von der politischen zur kulturellen Hegemonie, en particulier p. 90-100.

${ }^{15}$ Cf. BABEL, Deutschland und Frankreich im Zeichen der habsburgischen Universalmonarchie. 
Comme l'a très bien montré Jörg Ulbert ${ }^{16}$, la perception classique d'une augmentation des pouvoirs constitutionnels de l'empereur ressentie comme un danger, soit imminent, soit potentiel, menaçant le royaume de France avait survécu à l'époque où un tel péril avait été réel ou du moins plausible. À certains égards, et c'est particulièrement vrai pour la condition attribuée à l'empereur, des stéréotypes s'étaient formés qui faisaient preuve d'une grande longévité.

Si la perception française de la Constitution impériale suivait souvent de près les évolutions et les débats qui avaient lieu dans l'Empire, tous les acteurs n'étaient pas à l'abri de préjugés. On constate que, malgré toutes les sources d'information dont disposaient leurs compatriotes restés en France, les Français qui se trouvaient sur place, en Allemagne, avaient de meilleures chances de comprendre les évolutions les plus récentes.

L'évolution de l'image du Saint-Empire chez les Français, de 1643 à 1756, n'a pas été uniforme. Par conséquent, on devrait s'abstenir de toute tentative d'explication téléologique. Il n'y a pas de voie unique qui mène d'une perception prévalente de l'Empire comme un sÉtat< fédéral à caractère mixte - dans la deuxième moitié du XVIIe siècle - en passant par la négation de son caractère étatique chez certains auteurs au milieu du XVIII siècle - au rôle actif que la France napoléonienne prit dans la dissolution du Saint-Empire, en 1806. Au XVIIe siècle, il y avait déjà des auteurs français qui considéraient les princes de l'Empire comme quasi souverains; au milieu du XVIII ${ }^{e}$, certains auteurs attribuaient encore aux éléments monarchiques de la Constitution impériale une importance majeure. Or, à côté des persistances, il y avait aussi les retours en arrière, qui peuvent parfois s'expliquer par des évolutions dans l'Empire qui redonnaient une certaine actualité à des auteurs tels que Bodin, Chemnitz ou Pufendorf.

Contrairement aux études récentes qui ont souligné le caractère limité des connaissances des Italiens sur l'Allemagne et la Suisse ${ }^{17}$, nous sommes donc arrivé à des résultats positifs quant à l'état des connaissances que les Français d'Ancien Régime avaient de l'Empire. Pourtant, notre étude a aussi montré des carences.

En effet, s'il y a un aspect particulier qui apparait très clairement dans la majorité des sources que nous venons d'étudier, c'est la tendance des Français à interpréter l'ordre politique du Saint-Empire d'une façon dichotomique, en opposant d'un côté les états et de l'autre l'empereur. Or, cette dichotomie simplifie les réalités constitutionnelles de l'Allemagne. Les clivages politiques, religieux, constitutionnels, dynastiques, etc. ne manquaient pas entre les états, et on peut même prétendre qu'un élément structurel de ce système résidait dans le fait que certains ordres, dans ce genre de conflits, cherchaient l'appui et le soutien de l'empereur; ce dernier jouait aussi un rôle protecteur face aux petites principautés et à une grande partie des villes impériales soucieuses de garder leur indépendance par rapport aux territoires princiers qui les entouraient. Au lieu d'une image de l'Em-

16 Cf. notamment UlBERT, Frankreichs Deutschlandpolitik.

17 Bilan contradictoire dans: Alexander Koller (dir.), Die Außenbeziehungen der römischen Kurie unter Paul V. Borghese, Tübingen 2008 (Bibliothek des Deutschen Historischen Instituts in Rom, 115). 
pire perçu comme une espèce de république aristocratique, vision que l'historiographie traditionnelle du XIX ${ }^{e}$ et du début du XX ${ }^{e}$ siècle a maintes fois reprochée aux Français d'Ancien Régime, mais qui en fait n'avait pas toujours été majoritaire parmi eux, nous pensons que le véritable problème des Français était de se dégager de cette conception dichotomique; s'ils ont bien fait des progrès à ce propos au XVIIIe siècle, s'en tenir à une telle dualité fut peut-être l'erreur fondamentale du siècle de Louis XIV à l'égard de l'Allemagne.

Certes, il est problématique de parler indistinctement des »Français« au pluriel. Résumons ce qu'on vient de dire, dans les différents chapitres, au sujet des personnes qui se sont intéressées en France au droit public d'Allemagne, et reprenons ce qu'on a dit à propos de la question de savoir pourquoi les Français s'y sont intéressés et à quels moments ils l'ont fait: à en juger par les papiers que l'on peut retrouver de nos jours dans les archives et dans les cabinets de manuscrits des bibliothèques, au début de la période étudiée, vers 1648 , seulement quelques dizaines de Français avaient probablement une bonne connaissance du droit public du Saint-Empire; on peut supposer que quelques centaines de personnes devaient en avoir des connaissances approximatives. Parmi les bons connaisseurs, il y avait les professionnels de la diplomatie et les savants: le comte d'Avaux, durant plusieurs décennies ambassadeur français en Allemagne, et, à la fin de sa carrière diplomatique, ambassadeur plénipotentiaire au congrès de Münster; les Godefroy, surtout Théodore Godefroy, conseiller juridique de la délégation française; les Dupuy, érudits qui travaillaient sur l'histoire et sur le droit des différents États de l'Europe, etc. Il est difficile de dire de quelles connaissances disposait le personnel de ces diplomates et de ces savants; par exemple, contrairement à son maître, le secrétaire de Théodore Godefroy, Nicolas Doulceur, n'a guère laissé de traces qui puissent témoigner de sa propre vision du Saint-Empire, mais sa fonction exigeait évidemment de lui certaines notions de l'histoire et du droit d'Allemagne. Cependant, notre étude de la politique française au congrès de Westphalie nous a incité à conclure que la diplomatie française n'a pas toujours obtenu les bons résultats que la France a effectivement remportés dans le traité de Münster, et qui sont indéniables, à cause de la bonne connaissance du pays et d'un programme bien défini, mais parfois malgré certaines lacunes et en dépit de l'absence d'une vision d'ensemble de sa politique allemande ${ }^{18}$. À part les diplomates, d'autres fonctionnaires devaient entretenir des contacts plus ou moins réguliers avec des institutions allemandes, entre autres les militaires.

18 Ce résultat qui contredit dans une certaine mesure la position des sciences politiques, pour lesquelles une politique couronnée de succès est impossible sans avoir de connaissances très précises du pays avec lequel on négocie, s'accorde en revanche parfaitement avec les résultats que Wolfgang REINHARD a obtenus en étudiant la politique de la curie romaine: en effet, celui-ci conclut que malgré l'image parfois caricaturale que le Saint-Siège avait par exemple de l'Allemagne et de la Suisse, il arrivait à défendre avec succès ses intérêts dans ces pays; cf. ID., Historische Anthropologie frühneuzeitlicher Diplomatie: Ein Versuch über Nuntiaturberichte 1592-1622, dans: ROHRSCHNEIDER, STROHMEYER (dir.), Wahrnehmung des Fremden, p. 53-72. 
Parmi les personnes qui ont eu, dans la deuxième moitié du XVIIe siècle, une certaine connaissance du droit public allemand, on peut compter, outre les professionnels, une partie des élites du pays: juristes cultivés, nobles passionnés d'histoire, etc. Pourtant, si une partie des traités du droit public allemand furent écrits pour servir à l'éducation de certains jeunes nobles, il n'est pas certain qu'ils aient véritablement été utilisés à cet effet. Aucune des sources que nous avons consultées ne permet de tirer des conclusions sans appel au sujet du nombre de personnes qui professaient un intérêt pour le droit et l'histoire d'Allemagne. À en juger par les tirages moyens de la »Gazette de France « et des livres d'histoire dans la seconde moitié du XVII' siècle, il devrait s'agir de quelques centaines de personnes. Tout au plus quelques dizaines d'entre elles disposaient probablement d'une bonne connaissance du droit et de l'histoire de l'Allemagne. Rarissimes étaient celles qui étaient capables d'écrire des mémoires et des livres sur ces sujets. Cela explique le succès que certains Allemands et surtout que certains Alsaciens, qui comblaient cette lacune, ont connu dans la deuxième moitié du XVIIe siècle.

Le fait que la France fît appel à ces Allemands et aux Alsaciens pour se voir renseigner sur le droit public allemand n'était pas le résultat d'une politique réfléchie et systématique de la part du gouvernement français. Certes, nous disposons parfois de documents qui attestent bien qu'une démarche du gouvernement fut à l'origine, par exemple, de l'emploi d'une certaine personne. Cela est d'autant plus considérable que la curie romaine, autant que nous sachions, évitait justement d'employer des Allemands dans ses relations avec l'Empire, malgré leurs compétences, parce qu'on les soupçonnait de poursuivre leurs propres intérêts dans leur pays d'origine. Mais l'institutionnalisation, qui se traduisit notamment par la création d'un poste d'expert pour le droit public allemand, fut seulement une affaire du XVIII' siècle. Au XVII e siècle, on agissait en général en cas de nécessité, quand une mission était à accomplir ou un problème particulier à résoudre. Dans certains cas, ce n'était d'ailleurs pas une demande de Paris ou de Versailles mais une démarche personnelle qui était à l'origine d'un transfert de connaissances d'Allemagne en France: par exemple, Louis Du May, envoyé du duc de Wurtemberg, profita de ses négociations à Paris pour rendre public son ouvrage rédigé en français sur le droit public d'Empire; Jean Heiss offrit de son propre chef ses services au secrétaire d'État à la Guerre. Les ressortissants de l'Empire et les Alsaciens étaient donc conscients du besoin que les Français éprouvaient d'obtenir des informations sur l'Allemagne; pour ces Allemands et pour les Alsaciens concernés, c'était un moyen soit d'acquérir une notoriété en tant que savant, soit de se faire rémunérer pour les renseignements qu'ils pouvaient fournir.

En revanche, dans les années 1740 et au début des années 1750, les Français qui ont écrit des livres sur l'Empire sont déjà plus de douze, sans compter ceux qui ont rédigé des mémoires et dont le nombre est incertain, étant donné qu'une grande partie de ces mémoires sont anonymes. Si l'on trouve toujours des Allemands et des Alsaciens parmi les auteurs de livres français sur l'Empire, ce n'est plus par nécessité mais à cause de l'évolution culturelle qui avait fait du français 
la première langue d'Europe ${ }^{19}$. À ce moment-là, on peut estimer qu'il y avait plus de cent personnes en France qui disposaient de bonnes connaissances en la matière, si on inclut dans ce nombre, outre les auteurs d'ouvrages, les diplomates, les philosophes qui s'intéressaient à l'Allemagne, les universitaires et les savants qui entretenaient des relations avec leurs collègues allemands, etc. Au même moment, peut-être quelques milliers de personnes (lecteurs de ces livres d'histoire et de droit ainsi que des journaux) en avaient probablement une connaissance approximative. La connaissance approfondie du droit germanique ne faisait pas partie de la culture qu'on attendait d'un honnête homme, car il s'agissait d'un domaine technique; cependant, l'analyse que nous avons faite des mémoires de Saint-Simon, dans notre chapitre sur l'opinion publique et la connaissance du droit allemand, a montré que les milieux de la cour pouvaient avoir des notions précises dans cette matière. D'ailleurs, le fait qu'un nombre croissant de livres imprimés (comme ceux de Du May au XVII e siècle) et de manuscrits français (tel le traité de Lautz, au XVIII e siècle) sont destinés à l'éducation des jeunes princes montre que l'étude de l'histoire et des structures politiques du Saint-Empire entre progressivement dans la culture des élites.

Les Français s'intéressaient surtout à la forme du gouvernement de l'Empire en général, au statut de l'empereur, à la condition juridique des états d'Empire et à leur participation à la législation, au fonctionnement de la diète et aux élections impériales; ils accordaient une importance particulière aux lois fondamentales, d'abord à la paix de Westphalie: parmi les Français des XVII e et XVIII e siècles, il n'y avait guère d'historien qui ne fît allusion à la grande paix, dans un de ces "Abrégés chronologiques« tant à la mode du siècle ${ }^{20}$. C'est surtout à partir du règne de Charles VI que les Français s'intéressèrent à la capitulation impériale. Bien qu'ils portassent aussi un intérêt certain à la justice dans l'Empire, celle-ci ne comptait pas parmi leurs sujets de prédilection. En revanche, comparé à leurs connaissances dans ces domaines, ils s'intéressaient peu au droit de religion; surtout au XVIIe siècle, les textes français qui expliquaient bien les problèmes du droit religieux de l'Empire étaient assez rares. Malgré une certaine amélioration qu'on peut observer à cet égard au XVIII'e siècle, ce n'était certainement pas leur point fort.

Certes, sur le droit religieux comme sur les autres aspects du droit public de l'Empire, les Français pouvaient consulter, non seulement les ouvrages et les mémoires rédigés en français, mais aussi les publications en latin des juristes alle-

19 En outre, l'Alsace fait vraiment partie du royaume, à ce moment-là, de sorte que, malgré certains attachements culturels qui continuaient à lier d'une manière particulière les Alsaciens à la civilisation allemande, il faut désormais compter les Alsaciens parmi les Français. ${ }^{20} \mathrm{Cf}$, par exemple, [François Eudes] MÉzeray, Abregé chronologique de l'histoire de France, nouvelle édition augmentée. Tome douzième [...], Amsterdam 1755, p. 328-334; [MAuberT DE Gouvest], Histoire politique du siècle, p. 13-26; [M.] D'AvrignY (S.J.), Mémoires pour servir à l'histoire universelle de l'Europe, depuis 1600. jusqu'en 1716 [...]. Nouvelle édition corrigée et augmentée. Tome troisieme, 1648-1675, Paris 1757, p. 17 et 27-34. 
mands (les ouvrages rédigés en langue allemande échappaient à la majorité des Français - comme à la majorité des Français cultivés - étant donné que peu d'entre eux savaient lire l'allemand). Si les écrits des auteurs allemands étaient connus en France, à en juger par les citations qui en étaient faites dans les livres français et dans les mémoires manuscrits, et s'ils y étaient notamment propagés par les Alsaciens, auprès desquels le secrétaire d'État aux Affaires étrangères demandait par exemple des renseignements sur les juristes et les historiens allemands à consulter en matière de droit public, beaucoup d'indices, par exemple les citations dans les ouvrages et les mémoires ainsi que les notes de lecture conservées dans plusieurs cabinets de manuscrits, montrent que les Français d'Ancien Régime préféraient apparemment la lecture d'ouvrages en français au détriment du latin, une tendance qui s'accentua au cours du XVIII' siècle. Dans la mesure où le français s'établit comme la première langue des diplomates et des savants, non seulement les auteurs français, mais aussi des historiens et des juristes d'autres nations, et même certains Allemands, commencèrent à écrire en français sur le droit public du Saint-Empire; contrairement aux auteurs de la deuxième moitié du XVIIe siècle, ils ne visaient plus un public exclusivement ou majoritairement composé de Français.

C'était donc en français que les ressortissants du royaume cherchaient à comprendre l'Empire, et c'était dans cette langue qu'ils le décrivaient; en effet, si de Thou avait encore écrit en latin sur l'histoire du Saint-Empire, il n'y a, en règle générale, plus d'écrits latins sur ce sujet après la paix de Westphalie. Contrairement à l'Allemagne, la France ne disposait donc pas d'une propre littérature latine sur le ius publicum Imperii. C'était en français que les diplomates français envoyés en Allemagne rédigeaient leurs dépêches, recevaient leurs instructions et menaient une grande partie de leurs négociations; $c$ 'était en français que les juristes, les historiens et les employés du dépôt des archives écrivaient leurs ouvrages et leurs mémoires; enfin, c'était aussi en français que des savants comme le père Joseph Barre correspondaient avec leurs collègues allemands et discutaient des problèmes du droit public de l'Empire (bien que le latin jouât encore un certain rôle dans la correspondance épistolaire entre les savants, les progrès du français dans ce domaine étaient considérables durant la période étudiée).

Selon les résultats de nos recherches sur l'emploi des langues, le latin fut en revanche utilisé plus longtemps dans les négociations politiques que l'on n'a tendance à le croire généralement. Il reste dominant au siècle de Louis XIV, malgré le rayonnement de la culture française à cette époque-là, et garde encore son importance pour les documents solennels, tels les traités de paix, au milieu du XVIII' siècle; mais à ce moment-là, un observateur lucide, Frédéric-Charles Moser, s'aperçut du fait que le français était en train de l'emporter définitivement sur le latin.

Nous avons vu que le latin ne perdit pas son importance dès le XVIIe siècle faute de pouvoir traduire les idées du monde moderne, comme certains historiens et philologues l'ont prétendu. Théodore Godefroy, qui était un bon philologue et surtout un très bon juriste, préconisa dans les années 1640 l'emploi du latin dans 
les négociations franco-allemandes parce que le latin lui paraissait justement être la langue la plus propre à définir les problèmes juridiques dont on devait discuter. Pour prendre le relais du latin dans les relations franco-allemandes, le français devait développer une terminologie propre à traduire les particularités juridiques de l'Allemagne. Notre analyse des traductions françaises des traités et des lois fondamentales du Saint-Empire a montré que dès les années 1680 le français dispose de mots pour traduire presque tous les termes du droit public allemand.

En ce qui conceme les problèmes terminologiques que posaient ces traductions, nous avons insisté sur l'importance de l'interprétation correcte du concept de "souveraineté«, utilisé dans certaines sources dans le sens de Landeshoheit et dans d'autres, en revanche, dans le sens de "souveraineté absolue «. Étant donné que l'historiographie des $\mathrm{XIX}^{\mathrm{e}}$ et $\mathrm{XX}^{\mathrm{e}}$ siècles a souvent jugé la vision française de la condition juridique des États territoriaux allemands sur l'utilisation de ce concept, il s'est avéré nécessaire de déterminer ce que les Français d'Ancien Régime entendaient par ce mot, en parlant de la souveraineté des princes d'Empire, pour vérifier si les conclusions qu'on a tirées sur leur vision de l'Empire étaient exactes.

Nous avons pu constater que par les termes de »souveraineté« et de »droits de souveraineté «, les Français des XVII et XVIII ${ }^{e}$ siècles entendaient non seulement la souveraineté d'une tête couronnée qui ne dépendait que de Dieu et de son épée, mais aussi les droits dont un prince de l'Empire jouissait en vertu de sa Landeshoheit; les »droits de souveraineté « n'étaient pas toujours synonymes de la souveraineté dans le sens de »souveraineté absolue «21. Si nous avons considéré surtout la période qui va de 1643 à 1756 , l'ambiguitté du mot »souveraineté« en français n'est pas une particularité de ce siècle. Ce double usage est encore attesté dans le français des XIX et $\mathrm{XX}^{\mathrm{e}}$ siècles. Dans la première moitié du XVIIe siècle, les positions divergentes sur ce concept sont incarnées par Le Bret et Loyseau. Ce dernier estime que le souverain doit »ne tenir que de Dieu et de l'épée«; pourtant, »le Prince feudataire ne laisse d'être souverain, bien que sa souveraineté ne soit ni si excellente, ne si parfaite que celle qui ne relève d'aucun«. Pour Loyseau, non seulement les ducs, comtes et princes de l'Empire sont souverains, mais aussi ceux de France 22 . Cardin Le Bret soutenait par contre que la souveraineté était inséparablement attachée à la royauté: »on ne doit attribuer le nom et la qualité d'une souveraineté parfaite et accomplie, qu'à celles qui ne dépendent que de Dieu seul et qui ne sont subjectes qu'à ses loix «²3. Selon Roland Mousnier, »Le

${ }^{21}$ Signalons que l'Allemand Kreittmayr, ministre principal de la Bavière au XVIIIe siècle, tout en ne cherchant point à faire de son pays un État souverain, estimait que les territoires de l'Empire jouissaient de tous les »droits de souveraineté « (»Souveränitätsrechte«), cf. Wolfgang QUINT, Souveränitätsbegriff und Souveränitätspolitik in Bayem. Von der Mitte des 17. bis zur ersten Hälfte des 19. Jahrhunderts, Berlin 1971 (Schriften zur Verfassungsgeschichte, 15), p. 70.

22 Charles LoYseau, Traite des seigneuries [...]. Edition troisieme, corrigee et augmentee par l'autheur, Chasteaudun 1610, II, p. 43 et 94.

${ }^{23}$ Car[din] LE BrET, De la souveraineté du Roy [...], Paris 1632, I, p. 2. 
Bret dépassait Jean Bodin et esquissait la théorie du pouvoir totalitaire d'un gouvernement de guerre $\ll^{24}$.

Certains mots français ont par la suite pris un sens plus large en français, et sont définitivement entrés dans la langue de Molière: c'est par exemple le cas du terme »immédiateté«. D'autres mots n'ont pas dépassé le langage des spécialistes, comme par exemple »réversales «25. Certains termes, comme »supériorité territoriale«, semblent avoir été généralement acceptés au XVIIIe siècle, mais ont depuis perdu leur place dans la langue française, abstraction faite du langage des historiens spécialistes du droit public du Saint-Empire. Nous n'avons repéré que très peu de cas où les auteurs de langue française n'ont jamais trouvé de traduction unanimement acceptée; c'est le cas du terme allemand »kreisausschreibender Fürst «, pour lequel il y avait des traductions françaises, comme »prince convocateur «, mais si peu connues que les auteurs se sentaient obligés en règle générale de citer le terme allemand qu'ils voulaient traduire.

Quant au concept de »supériorité territoriale «, nous avons constaté qu'il n'a été considéré comme faisant vraiment partie de la langue française qu'au XVIII' siècle; s'il existe depuis le XVII e, les auteurs, surtout ceux des ouvrages destinés à être imprimés, hésitent à le reprendre, tandis que les états d'âme à cet égard diminuent sensiblement au siècle suivant. Dans la période que nous avons étudiée, nous avons trouvé un nombre non négligeable d'auteurs qui s'en servent. Ce concept avait sa place dans les écrits où il était indispensable pour éviter toute équivoque, c'est-à-dire surtout dans les négociations avec l'Empire et dans les instructions fournies aux diplomates ${ }^{26}$. Quand il est employé dans les ouvrages imprimés, il apparaît toutefois, surtout au XVII ${ }^{e}$ siècle, comme un corps étranger, même dans le langage des juristes et des historiens, qu'il n'a jamais dépassé. Les auteurs qui parlent de "supériorité territoriale «se croient le plus souvent obligés d'expliquer ce terme, de le préciser par son équivalent allemand, ou, ce qui se fait encore aujourd'hui, de le mettre en italique ou entre guillemets.

Or, le fait que ce concept existe a amené certains historiens, en particulier ceux d'outre-Rhin, à prétendre que tous les auteurs qui parlent de »souveraineté« à l'égard des ordres n'auraient pas bien compris les données constitutionnelles du Saint-Empire, d'où ils ont déduit un manque de connaissances des Français sur ce sujet. Les travaux de certains historiens, notamment de Klaus Malettke, ont toutefois prouvé que l'on a longtemps sous-estimé les connaissances profondes des di-

24 MousniER, Les institutions de la France, t. I, p. 514.

25 Pour l'importance des mots étrangers dans le langage juridique de l'époque moderne, cf. aussi Andreas GöRGEN, Rechtssprache in der Frühen Neuzeit. Eine vergleichende Untersuchung der Fremdwortverwendung in den Gesetzen des 16. und 17. Jahrhunderts, Francfort/M. 2002 (Rechtshistorische Reihe, 253).

26 Charles François Lefèvre DE LA MAILlaRDière, Histoire politique de l'Allemagne, et dès Etats circonvoisins, dépendances anciènnes ou actuèlles de l'Empire [...], Paris 1777 (ouvrage destiné à l'instruction des négociateurs): 》[La paix de Westphalie] établit solidement et la supériorité tèrritoriale dès Etats dans leurs Provinces, et leurs droits comitiaux, en lès constituant co-Régens de l'Empéreur dans tout ce qui concerne l'administration dès affaires publiques de l'Allemagne [sic]« (p. 102-103). 
plomates et des juristes français ${ }^{27}$ dans cette matière si difficile et discutée parmi les Allemands eux-mêmes ${ }^{28}$. Il est incontestable que certains auteurs français qui ont écrit entre 1643 et 1756 ont réellement tenu les États territoriaux pour véritablement souverains; cependant il ne suffit pas, pour en arriver à cette conclusion, de constater qu'ils emploient le terme de »souveraineté« à l'égard des princes, puisque celui-ci est ambigu et que les auteurs qui défendent une autre vision de l'Empire y recourent aussi.

Cependant, l'ambiguitté du terme »souveraineté « crée un certain malaise. Il se fait encore sentir quand Goubert parle, dans son »Mazarin«, d'une »théorique indépendance « des états de l'Empire après $1648^{29}$. Le français manque d'un concept clair pour désigner un phénomène intermédiaire entre souveraineté et dépendance. S'il y a »indépendance « des princes, celle-ci est essentiellement pratique, non pas juridique. Les états de l'Empire ne menèrent d'ailleurs aucunement, au milieu du XVIIe siècle, une politique >souverainiste ‘; l'accession au statut d'États indépendants ne faisait pas partie de leurs objectifs politiques ${ }^{30}$. L'étoile de la souveraineté bodinienne, qui s'était levée dans le firmament du langage politique français à la fin du XVIe siècle, transcenda la terminologie de la féodalité sans jamais l'éteindre. Dans la sphère de la langue, il n'y a pas la succession de l'État souverain au monde médiéval, enseignée par Georges Pagès dans sa grande synthèse ${ }^{31}$, mais une coexistence de deux systèmes provenant d'époques différentes, avec toutes les ambivalences qu'elle implique.

D'une manière générale, en ce qui concerne la forme de gouvernement du Saint-Empire, les idées divergentes des historiens et des juristes français des $\mathrm{XVII}^{\mathrm{e}}$ et XVIII ${ }^{\mathrm{e}}$ siècles ne sont pas liées à l'emploi d'un vocabulaire spécifique. Tous partent, par exemple, de traductions presque identiques des traités de Westphalie et des autres lois fondamentales de l'Empire ${ }^{32}$. Les idées ne sont donc pas attachées aux mots, mais aux notions. Outre les termes de »souveraineté« et de »supériorité«, ce sont surtout la capitulation impériale et le ban de l'Empire qui

${ }^{27} \mathrm{Cf}$. les nombreuses contributions de MaLETTKE que nous avons citées tout au long de cet ouvrage et que l'on peut retrouver très facilement dans la bibliographie.

28 Voltaire écrit à propos du droit public allemand: "Chaque membre de l'empire a ses droits, ses priviléges, ses obligations; et la connaissance difficile de tant de lois, souvent contestées, fait ce que l'on appelle en Allemagne l'étude du droit public, pour laquelle la nation germanique est si renommée«, VolTaIRE, Le siècle de Louis XIV, édition Paris 1994, p. 7. 29 Cf. Pierre GouberT, Mazarin, Paris 1990, p. 164-184.

${ }^{30}$ Cf., par exemple, l'ouvrage de Quint sur la Bavière: Maximilien Ier, qui menait une véritable politique étrangère au niveau européen et qui régnait en prince presque absolu sur la Bavière comme ne le faisaient que peu de princes allemands sur leurs territoires, ce prince très puissant ne réclama jamais le titre de »souverain«. II ne suivit point les »Six livres« de Bodin, mais se laissa guider par la »ragion di stato « de Juste Lipse, QUINT, Souveränitätsbegriff, p. 53-61.

31 PaGÈs, La guerre de Trente Ans.

32 Cette conclusion est incontestable pour les auteurs qui publient des versions françaises de ces lois fondamentales à la fin de leurs livres sur l'Empire; malgré des versions presque identiques, ils en tirent des conclusions divergentes, parfois même contraires, quant à la condition de l'empereur et des états. 
sont soumis à des interprétations divergentes. Malgré ces discordances, les historiens, les juristes et les diplomates français ne voient généralement en la paix de Westphalie qu'un élément décisif parmi tous ceux qui ont formé la Constitution du Saint-Empire; un auteur comme Bruneau, qui pense que les traités de 1648 ont stipulé la souveraineté des princes, fait plutôt figure d'exception. Les sindépendantistes $<-$ si on veut permettre l'emploi de ce terme pour désigner ceux qui considèrent les ordres comme largement indépendants de l'Empire - se fondent en particulier sur les capitulations impériales. D'autres historiens, qui s'abstiennent largement de jugements idéologiques, comme Pfeffel, parlent, à l'égard de la capitulation, d'une "confirmation « des droits des ordres, tout en reconnaissant la paix de Westphalie comme le "Code politique et la principale des loix fondamentales de l'Empire Germanique ${ }^{33}$.

En ce qui concerne les traités de Westphalie, toutes les traductions sont, des points de vue terminologique et stylistique, très proches de l'original latin. Seules les premières traductions comportent certaines fautes surprenantes au niveau de la terminologie; à partir de Heiss, on ne trouvera plus guère de différences majeures. Si l'on peut constater que les autres traités de paix passés entre la France et l'Empire et les capitulations impériales ainsi que la Bulle d'or sont aussi, en règle générale, assez bien traduits en français, force est de constater que l'on arrive à des conclusions plus mitigées quant aux clauses religieuses de 1552 et de 1555 . Toutefois, il faut noter qu'à la fin du XVIIe siècle les dénominations des institutions du Saint-Empire, à quelques exceptions près, sont celles qu'on emploie encore aujourd'hui. Il semble bien qu'elles aient été développées à partir de la terminologie latine.

Finalement, on peut conclure que la terminologie française développée pour décrire la Constitution allemande était parfaitement capable d'accomplir sa tâche et qu'il serait faux d'insinuer qu'elle n'a point existé. L'emploi du français dans certains traités passés entre les princes allemands au milieu du XVIIIe siècle ${ }^{34}$, par exemple entre l'Autriche et la Prusse, présuppose en effet l'existence d'une terminologie propre à exprimer la volonté des parties contractantes et les réalités constitutionnelles et juridiques dont il s'agissait de convenir; autrement, ces parties n'auraient certainement pas conclu leurs traités en français, mais en allemand ou en latin.

Le latin, quant à lui, était toujours capable d'assumer cette tâche et il fut effectivement employé pour d'autres traités conclus à la même époque. Brunot a donc tort, à cet égard, s'il juge que "malgré la Renaissance et aussi à cause de la Renaissance, le latin avait cessé d'être apte à vivre de la vie des nations modernes. Restauré dans sa pureté antique, il était devenu en effet par cela même à peu près incapable d'exprimer certaines idées modernes, scientifiques ou politiques « 35 . Le fait que le droit international (il n'y a qu'à voir Grotius) et la Reichspublicistik

33 Pfeffel, Abrégé chronologique (édition de 1754), p. 530-532.

34 Cf. ScheId, Traité sistématique, t. I, p. [*4'].

35 BRUNOT, Histoire, t. V, p. 388-389. 
s'expriment d'abord presque exclusivement en latin, et qu'encore en 1751 il est jugé indispensable de savoir le latin et l'allemand pour »acquérir une connoissance exacte de toutes les matières du droit allemand « ${ }^{36}$, prouve le contraire; les idées modernes naissent et s'expriment dans la langue ancienne. Ce n'est qu'en acquérant les mêmes capacités que le français a pu devenir la langue de la diplomatie et des sciences politiques. Au milieu du XVIII siècle, c'est un fait acquis.

Le nombre de traductions de traités internationaux et de lois fondamentales que nous avons trouvées atteste la prédilection des Français pour les traités de Westphalie; d'abord discret, jusqu'aux années 1680 , l'intérêt qu'on porte à ces traités ne diminue guère jusqu' au milieu du XVIII' siècle, bien que les capitulations impériales gagnent du terrain depuis Charles VI. De toute façon, les traductions des traités de Westphalie sont de loin plus nombreuses que celles des autres lois fondamentales, des capitulations et des traités de paix. Il était surtout difficile de trouver, en France, une bonne traduction du traité de Passau et de la paix de religion d'Augsbourg.

Certes, on ne traduisait pas en français seulement les lois et les traités, mais aussi les ouvrages de certains juristes et de plusieurs historiens: nous avons vu que l'ouvrage »De Statu Imperii Germanici« de Samuel Pufendorf a été traduit en français à plusieurs reprises, de même que la »Dissertatio de Ratione Status «par Chemnitz; ces auteurs du XVIIe siècle ont par ailleurs continué à être traduits en français au XVIII ${ }^{e}$. On a aussi traduit le »Corpus iuris publici« de Schmauss et la "Geschichte der Deutschen « de Schmidt. À part ces traductions imprimées, il y a aussi des versions françaises manuscrites: on peut citer celle de Jean-Jacques Moser conservée à la Bibliothèque nationale de France ${ }^{37}$.

Or, depuis le XVIIe siècle, les auteurs français et de langue française qui ont écrit sur le droit et l'histoire de l'Allemagne ne manquent pas, et au XVIII', ils sont de plus en plus nombreux: Le Secq, Wicquefort, Bruneau, Du May, Du Val, Heiss, Prade, Rocoles, Varillas, Courtalon, Du Buat-Nançay, Fréron, Gérard de Rayneval, Jacquet, La Maillardière, Le Coq de Villeray, Massuet, Mauvillon, Montigny, Necker, Pfeffel, Rousset, Scheid, Schmettau, Spon, Vayrac et Voltaire sont des auteurs avec des approches juridiques, historiques et philosophiques différentes et qui témoignent de la diversité des visions de l'Empire qui ont existé en France. L'auteur le plus influent fut certainement Jean Heiss, qui domina pendant cinquante ans le marché du livre en France, avec les neuf éditions de son »Histoire de l'Empire«, publiées entre 1684 et 1733; pourtant, ses idées ne furent point unanimement acceptées, témoin les notes de bas de page que Bourgeois du Chastenet ajouta à son édition de l'ouvrage de Heiss et dans lesquelles il dit souvent le contraire de ce que Heiss explique dans son texte. Un auteur français des années 1740 (Joseph Barre) fut même traduit en allemand, ce qui atteste l'essor de la littérature française sur l'histoire du Saint-Empire. Cependant, l'ouvrage systématique d'un

36 Cf. ScheId, Traité sistématique, t. I, p. [ $\left.{ }^{*} 8^{\prime}\right]$.

37 BNF, F.fr. 12113. 
Allemand (Scheid) et l'ouvrage historique d'un Alsacien (Pfeffel) marquent, en 1754, l'apogée de cette littérature française sur le droit public du Saint-Empire.

En ce qui concerne les manuscrits, nous avons constaté l'hétérogénéité des fonds conservés dans les bibliothèques, qui sont surtout composés de papiers de savants et de documents diplomatiques. Mais cette diversité traduit la panoplie des intérêts que les Français portaient à la Constitution du Saint-Empire et qui ne se limitaient point aux seuls professionnels de la diplomatie ou au monde universitaire. Quant au fonds des »Mémoires et documents« du Quai d'Orsay, qui a été dépouillé de manière systématique, l'auteur le plus actif a certainement été le premier commis du dépôt, Nicolas-Louis Le Dran, bien que l'on puisse formuler, dans plusieurs cas, des réserves sur l' attribution de certains documents. Quoi qu'il en soit, ce fonds est assez hétérogène: il n'est pas vrai qu'il comporte uniquement des documents issus des travaux de l'académie de Torcy; on peut y trouver de nombreux mémoires qui sont issus du travail mené au dépôt des Affaires étrangères et par les diplomates français en mission en Allemagne. Le point commun de tous ces documents est d'avoir joué un rôle dans la diplomatie française: soit dans le cadre de la formation des futurs diplomates, soit pour informer des envoyés, des hommes politiques ou le roi de certaines particularités du droit public de l'Empire dans une situation politique où de telles connaissances étaient indispensables. Pour cette raison, certains mémoires mentionnent au début le fait qu'une demande de renseignements de la part du gouvernement était à l'origine de leur rédaction. Par conséquent, le dépôt des archives ne conservait pas seulement les correspondances et les autres documents diplomatiques concernant la politique étrangère de la France dans le passé, constituant ainsi la mémoire de la diplomatie française, mais il offrait aussi un moyen de préparer la politique future, puisqu'on défrichait ces documents, souvent à l'instigation d'hommes politiques ou de diplomates, pour trouver des réponses à des demandes de renseignements d'ordre juridique ou politique.

Nous avons pu constater que, à partir de 1740 environ, le nombre de livres imprimés et de mémoires manuscrits traitant de l'histoire et du droit d'Allemagne augmente considérablement, de sorte que presque la moitié des documents que nous avons étudiés ou pu repérer datent de la période 1740-1756, l'autre moitié de celle qui va de 1643 à 1739 . De la paix de Westphalie à la fin des années 1730, on peut observer une progression continue mais assez nette du nombre des écrits français sur ces sujets. D'un côté, cette évolution peut s'expliquer par l'essor de l'imprimerie et de la publication d'ouvrages historiques et juridiques en général, qui est un phénomène culturel bien connu de cette époque-là, ainsi que par les progrès dans le domaine de la constitution des archives: il ne faut pas oublier que les mémoires rédigés au XVII e siècle avaient une plus grande chance de se perdre que ceux qui furent rédigés au XVIIIe; à cela s'ajoute l'institutionnalisation bureaucratique dans le domaine de la diplomatie et dans l'organisation respectivement du dépôt et du ministère des Affaires étrangères, qui entraînait une augmentation du nombre de documents que leur activité produisait. Or, de l'autre côté, la croissance de la production de documents à partir de 1740 est aussi très certaine- 
ment due aux évolutions politiques et constitutionnelles dans le Saint-Empire: en 1740, la crise de la succession à l'Empire est ouverte; les Habsbourg perdent temporairement la dignité impériale; l'essor de la Prusse, qui s'accentue durant le règne de Frédéric le Grand, change la donne constitutionnelle: désormais, un prince de l'Empire égale l'empereur en puissance. Certes, ces évolutions politiques qui ont marqué les années 1740 et 1750 et qui ont entraîné le Renversement des alliances sont déjà bien connues par l'historiographie des $\mathrm{XIX}^{\mathrm{e}}$ et $\mathrm{XX}^{\mathrm{e}}$ siècles. Or, les résultats de notre propre étude mettent en garde contre toute tentative de minimiser leur importance; car le changement fondamental qu'elles causaient en modifiant la Constitution de l'Empire, sinon de iure, du moins de facto, fut déjà perçu par les contemporains. Les Français notèrent ce changement et s' interrogèrent sur l'avenir du Saint-Empire; c'est pour cela que le nombre d'écrits augmenta d'une façon si spectaculaire.

En ce qui concerne les idées que leurs auteurs professent, on peut observer que les mémoires conservés au dépôt des archives restent, contrairement aux livres imprimés ${ }^{38}$, attachés à des idées plus traditionnelles. Cela confirme les résultats que Jörg Ulbert a obtenus en étudiant l'image de l'Empire en France pendant la Régence (1715-1723) ${ }^{39}$. Pourtant, la diplomatie française n'était pas insensible aux évolutions en cours dans l'Empire, de sorte que malgré une plus grande prudence, on y tira finalement la conclusion que l'autorité de l'empereur en était sortie affaiblie et que les forces centrifuges avaient augmentét ${ }^{40}$. La diversité des idées que l'on peut saisir dans les écrits français sur l'Empire depuis 1740 s'explique très certainement par les interrogations sur l'évolution future, qui paraissait plus incertaine que jamais.

En considérant l'évolution de la vision française de la Constitution germanique de 1643 à 1756, nous avons pu constater que les Français se dégagèrent successivement des préjugés liés à la Constitution de leur propre royaume. Tout au long de cette période, ils gagnèrent en sensibilité face aux particularités du droit germanique. Nous avons aussi pu voir que, au lieu de diminuer, leurs incertitudes avaient plutôt tendance à augmenter, surtout depuis 1740. Mais au lieu d'un manque de connaissances, ces incertitudes étaient justement le résultat de l'accroissement de la sensibilité; on ne se contentait pas d'idées préconçues, déduites de la situation politique et de l'organisation juridique qu'on connaissait dans le royaume. La vision des Français de l'Allemagne était de moins en moins déterminée par leur pro-

38 Abstraction faite de certains auteurs qui perpétuaient une vision plus traditionnelle de l'Empire avec un empereur puissant à sa tête, comme le Français Le Coq et l'Allemand Scheid, qui écrivait en français.

39 Cf. ULBERT, Frankreichs Deutschlandpolitik.

40 Pour l'image de l'Empire chez les diplomates français à l'époque de la guerre de Sept Ans, cf. ExTERnBrink, Friedrich der Große, Maria Theresia und das Alte Reich; cf. également ID., Frankreich und die Reichsexekution gegen Friedrich II. Zur Wahrnehmung der Reichsverfassung durch die französische Diplomatie während des Siebenjährigen Krieges, dans: AsBaCH, MALETTKE, EXTERnBRINK (dir.), Altes Reich, p. 221-253. 
pre expérience. La diversification des idées est, dans cette perspective, le signe du progrès qu'ils avaient fait en cherchant à comprendre la Constitution de l'Empire.

Il est certain qu' une grande partie de l'historiographie française des XIX et $\mathrm{XX}^{\mathrm{e}}$ siècles a considéré le Saint-Empire comme une anarchie de princes territoriaux ou comme une confédération d'États pratiquement souverains et indépendants. L'historienne autrichienne Ingred Offenbeck a établi un florilège trop convaincant des idées de ce genre pour que l'on puisse encore en douter ${ }^{4 l}$. Mais à l'époque où cette conception du Saint-Empire prévalait effectivement en France, les historiens allemands eux-mêmes avaient une image plutôt négative du droit constitutionnel fédéral perennisé par les traités de Westphalie; la création de l'État-nation leur apparaissait comme le but de l'histoire d'Allemagne, et on considérait que le caractère fédéral de l'Empire avait empêché ou retardé la création d'un État unifié et puissant. Il en va de même pour la propagande: un tract distribué à des millions de soldats allemands durant la Seconde Guerre mondiale établit un parallèle entre les traités de Westphalie et celui de Versailles, stigmatisant la France comme coupable de l'anarchie qui en aurait résulté en Allemagne après 1648 aussi bien qu'après 1919 .

Or, en Allemagne comme en France, le Saint-Empire jouissait d'un plus grand prestige aux Temps modernes qu'à l'époque de l'État nation. À partir des années 1950, ce fut d'abord l'Allemagne qui revint à des idées plus justes quant à l'interprétation de la Constitution du Saint-Empire, suivie des historiens français, notamment de ceux qui étaient issus de l'école de Victor-Lucien Tapié. En France aussi bien qu'en Allemagne, la perception du Saint-Empire dépendait donc de la situation subjective des historiens, des juristes et des hommes politiques. Si cette constatation est évidente, elle n'est pas gratuite: car elle s'oppose à l'instrumentalisation de l'histoire, qui stipule, pour des raisons politiques ou idéologiques, des continuités où il n'y en a pas.

À travers les ouvrages français, on voit ainsi que l'Allemagne n'est nullement perçue comme »une république de princes, une vaste anarchie sous le protectorat de la France«, ainsi que l'a prétendu Salomon à l'égard de l'Allemagne telle qu'elle se présentait vers $1749^{42}$; du moins ce n'est point l'idée qui prévaut en France et chez les auteurs de langue française. Même Necker, un auteur du milieu du XVIIIe siècle très critique à l'égard de toute tentative de limiter les prérogatives et les libertés des ordres, ne considère point l'Empire comme une anarchie, et fait même preuve de patriotisme d'Empire (Reichspatriotismus). La littérature française sur le droit public allemand reflète donc parfaitement les contestations qui se sont élevées au sujet de l'interprétation de celui-ci en Allemagne même, sur l'échiquier politico-juridique.

Dans la mesure où le français devient la première langue d'Europe, cette littérature française dépasse même, au milieu du XVIIIe siècle, le cadre de la France et des auteurs allemands qui ont depuis longtemps écrit pour un public francophone;

41 Cf. OFFENBECK, Der Dreissigjährige Krieg.

${ }^{42}$ Salomon, Les Alsaciens employés au Ministère des Affaires étrangères, p. 452. 
mais les Allemands d'origine, propageant leur culture de l'État de droit à l'étranger, se sont aussi mis au service d'autres puissances, tel un Necker chez les Britanniques, et c'est du français qu'ils se servent maintenant même s'ils ne visent pas un public (uniquement) francophone. La littérature française sur l'Empire échappe ainsi au continent pour conquérir même l'Angleterre, en subissant parfois certains changements non mineurs dans la manière de percevoir l'Empire, mais, à peu près, sans mutations majeures au niveau terminologique. Au milieu du XVIII' siècle, la France fournit donc la grille conceptuelle selon laquelle l'Europe cherchait à comprendre la Constitution du Saint Empire romain germanique.

\section{Perspectives: la survie de l'empire}

Si l'année 1756 marque une césure dans les relations entre la France et l'Empire ainsi que dans la vision des Français de leur voisin allemand, le Saint Empire romain germanique continua à exister jusqu'en 1806 , pour succomber finalement à la politique allemande de Napoléon. La commémoration du bicentenaire du crépuscule du Saint-Empire incite les historiens à réfléchir, non seulement sur l'histoire du Saint-Empire même, mais aussi sur la survie de celui-ci dans l'imaginaire.

Qu'en est-il en France? La disparition du Saint-Empire y a-t-elle modifié l'idée de l'empire et de la dignité impériale en général? Cette question dépassant largement le cadre de nos propres recherches, nous nous limiterons à un seul exemple qui nous paraît éloquent. À la fin du XIXe siècle, Pradier-Fodéré, dans un manuel de droit diplomatique, conclut que la fin du Saint-Empire en 1806 marqua la fin de la supériorité des empereurs par rapport aux rois, tout en accordant aux empereurs un plus grand prestige et un »pouvoir moins limité « ${ }^{43}$; mais seulement la »dénomination« de la dignité impériale traduisait encore, à son avis, son caractère universel, sans qu'il admette aucune »portée pratique au point de vue de l'idée de primauté«. Quant à la notion d'»empire«, Pradier-Fodéré constate: »elle convient surtout à des États d'une très grande étendue territoriale, tels que sont la Russie, la Turquie, l'Autriche, qui tiennent sous leur domination des races et des nationalités diverses«. L'idée d'une domination supranationale est donc toujours attachée à la notion d'»empire«.

Mais l'aspect le plus curieux des observations de Pradier-Fodéré sur le concept d'»empire« (il écrivit, en 1899, non pas un ouvrage historiographique, mais un

43 »De nos jours, où les empereurs d'Allemagne, qui étaient considérés comme occupant le premier rang dans la chrétienté, ont cessé d'exister comme chefs de ce que l'on appelait le Saint-Empire, le titre d'empereur n'a plus aucune supériorité réelle sur celui de roi. Les monarques ne le regardent donc plus comme étant, à lui seul, une raison suffisante pour prétendre à une prérogative quelconque. Ce que l'on peut dire, c'est que, sans affirmer que le titre d'empereur ait conservé encore aujourd' hui plus de prestige que celui de roi, le pouvoir impérial est, en général, moins limité que le pouvoir royal«, PRADIER-FoDÉRÉ, Cours de droit diplomatique, t. I, p. 71-72. 
manuel destiné aux futurs diplomates!) est que l'auteur retrace la lignée de succession qui va du premier empereur romain, Auguste, en passant par Charlemagne et Otton le Grand, jusqu'aux derniers empereurs du Saint Empire romain germanique ${ }^{44}$ - un jeune diplomate qui, en lisant cet ouvrage, s'initia aux fondements de son métier, vers 1900 , apprit donc la théorie de la translatio Imperii, dont le bien-fondé ne fut pas remis en cause; le Saint Empire romain germanique lui apparaissait donc toujours comme la perpétuation de l'Empire romain de l'Antiquité.

Quoique la vision française du Saint-Empire ait profondément changé dans une grande partie de l'historiographie française des $\mathrm{XIX}^{\mathrm{e}}$ et $\mathrm{XX}^{\mathrm{e}}$ siècles, ainsi que nous l'avons appris par Offenbeck, on peut donc observer des continuités dans le savoir qui est transmis aux diplomates, à travers les manuels, en ce qui concerne l'histoire d'Allemagne et les fondements historiques des concepts »empereur «, »empire«, »Saint-Empire« et »Saint Empire romain « ${ }^{45}$, qui dépassent la période que nous avons étudiée, et qui vont au moins du XVII ${ }^{e}$ jusqu'à la fin du $\mathrm{XIX}^{\mathrm{e}}$ siècle ${ }^{46}$.

Pour autant, en 1899, le terme »empereur«, qui avait désigné les empereurs romains, byzantins et romains germaniques (sans parler de Napoléon Ier et de Napoléon III), n'est plus qu'un souvenir; sa grandeur passée garde encore sa place dans la mémoire de l'époque, mais son usage paraît désormais anachronique: »Le titre d'empereur a été considéré, dans le passé, comme désignant la plus éminente dignité. [...] Le titre d'empereur et la dignité impériale ne sont plus, de nos jours, qu'un souvenir du passé, conservé par la vanité des dynasties et des nations ${ }^{47}$.

Si la dignité impériale (abstraction faite d'une certaine renaissance due au bicentenaire du Premier Empire français) a depuis longtemps perdu de son attraction, sans s'effacer complètement, le fédéralisme qui a caractérisé le Saint Empire romain germanique a conservé ou regagné tout son attrait auquel les Français des $\mathrm{XVII}{ }^{\mathrm{e}}$ et XVIII ${ }^{\mathrm{e}}$ siècles n'étaient pas restés insensibles.

44 Cf. ibid., p. 70-71, n. 2 et p. $72-73$ (citations).

45 Les deux derniers termes sont définis par Pradier-Fodéré comme »l'empire d'Occident«, qui fut rétabli par Charlemagne, et comme l'»ancien empire d'Allemagne«, qui avait été considéré comme son successeur (ibid., p. 70-71, n. 2).

46 Nous ne pouvons pas offrir ici une analyse détaillée de l'histoire de ces concepts dans la deuxième moitié du XVIIIe siècle et au XIXe.

${ }^{47}$ Pradier-Foderé, Cours de droit diplomatique, t. I, p. 69 et 74. 